\title{
Elast PQ Ultrasound - a Noninvasive, Reproducible, Easily Performed Method and a New Era in Liver Fibrosis Assessment
}

\author{
H Rahman
}

\begin{abstract}
:
Liver fibrosis represents the repair mechanism in liver injury and is a feature of most chronic liver diseases. The degree of liver fibrosis in chronic viral hepatitis infection has major clinical implications and presence of advanced fibrosis or cirrhosis determines prognosis. Treatment initiation for viral hepatitis is indicated in most cases of advanced liver fibrosis and diagnosis of cirrhosis entails hepatology evaluation for specialized clinical care. Liver biopsy is an invasive technique and has been the standard of care of fibrosis assessment for years; however, it has several limitations and procedure related complications. Recently, several methods of noninvasive assessment of liver fibrosis have been developed which require either serologic testing or imaging of liver. Imaging based noninvasive techniques are reviewed here and their clinical use is described. Some of the imaging based tests are becoming widely available, and collectively they are shown to be superior to liver biopsy in important aspects. Clinical utilization of these methods requires understanding of performance and quality related parameters which can affect the results and provide wrong assessment of the extent of liver fibrosis. Familiarity with the strengths and weakness of each modality is needed to correctly interpret the results in appropriate clinical content.
\end{abstract}

A new technique called Elast PQ uses ultrasound shear wave elastography to provide a noninvasive, reproducible, easily performed method of assessing liver fibrosis. It can easily combine a routine ultrasound imaging exam of the liver anatomy with targeted tissue stiffness values, assess liver fibrosis in patient with clinically suspected disease even before abnormalities are detected with ultrasound imaging, evaluate and obtain a baseline stiffness value in patients with chronic liver disease, follow up patients under treatment to monitor progression, stabilization or regression of liver disease and help avoid the need for liver biopsies when elastography results are consistent with other clinical findings.

Both the prognosis and potential treatment of chronic liver diseases greatly depend on the progression of liver fibrosis, which is the ultimate outcome of chronic liver damage. Historically, liver biopsy has been instrumental in adequately assessing patients allows clinicians both to obtain diagnosis information and initiate adequate therapy. However, the technique is not exempt of deleterious effects. Multiple diagnostic tests have been developed for the staging of fibrosis using noninvasive methods, most of them in the setting of chronic hepatitis $\mathrm{C}$. The goal of this paper is to review available data on the staging and assessment of liver fibrosis with two methods: serum markers \& transient elastography (FibroScan).

Key words: Liver Fibrosis, Liver biopsy, Transient Elastography, FibroScan.

\section{Introduction:}

Diffuse liver diseases is one of the major health problems in the word. It can result from many causes, including viral hepatitis (Hepatitis B or Hepatitis C), non-alcoholic or alcoholic fatty liver diseases, autoimmune hepatitis, drug induced liver injury, primary biliary cirrhosis, and several other less frequent etiologies. It is estimated that 360 million and 180

1. Dr. Hafizur Rahman, MBBS, M Phil (Nuclear Medicine), Principal Medical Officer, Institute of Nuclear Medicine and Allied Science, Faridpur.

Address of correspondence :

Dr. Hafizur Rahman, MBBS, M Phil (Nuclear Medicine), Principal

Medical Officer, Institute of Nuclear Medicine and Allied Science,

Faridpur. Mob.: +88-01711235280,E-mail: mhrn08@yahoo.com worldwide are infected with viral hepatitis $\mathrm{B}$ and $\mathrm{C}$ respectively. Between 5000,000 and 7000,000 people die annually as a result of hepatitis $B$ virus infection, and more than 350,000 are estimated to die each year from Hepatitis C-related liver diseases ${ }^{1-3}$. Chronic liver damage results in hepatic fibrosis, characterized by an increase in extracellular matrix material produced by fibroblast like cells. This process results in liver fibrosis that can progress to cirrhosis with distortion of normal liver architecture and portal hypertension.

Both the prognosis and potential treatment of chronic liver diseases greatly depend on the progression of liver 
fibrosis, which represents the ultimate consequence of chronic liver damage. This is a dynamic situation where two extreme progresses collide: fibrogenesis and fibrolysis ${ }^{4}$. This entails an accumulation of collagen, as well as other proteins in the extracellular matrix, in the tissue. Progressive deposition of these substances eventually results in disrupted liver morphology, parenchymal function impairment, and ultimately portal hypertension and its related sequels.

In the last few years progress has been made in understanding the cellular and molecular mechanisms leading to fibrogenesis, particularly regarding the relevance of inflammatory mediators, apoptosis, and the role of hepatic satellite cells.

\section{Diagnosis and staging of liver diseases:}

Accurately staging the degree of liver fibrosis is extremely important to determine if antiviral therapy is appropriate, and to predict treatment outcome and malignant potential. With current drug therapy, early stage fibrosis may be reversible ${ }^{5}$.

The histologic evaluation of liver biopsies is carried out using scoring systems scoring that produce values for varies categories of inflammation (grade), and fibrosis (stage). There have several scoring systems, all categories similar features. In the assessment of chronic HCV hepatitis, the most reproducible scoring system is the Metavir. On the Metavir scoring system, liver fibrosis is evaluated semi-quantitatively and staged on a five point scale from 0-4 (Fo: Fibrosis absent; F1: enlarged fibrotic portal tract: F2: peri-portal or initial portal-portal septa but intact architecture; F3: architectural distortion but no obvious cirrhosis; and F4: cirrhosis).

The gold standard for diagnosis and staging of liver fibrosis has been liver biopsy in addition to being an invasive procedure with potential complications of bleeding and severe pain, sampling error is an intrinsic problem due to the small sample size in a heterogeneous process ${ }^{6-7}$. Inter-observer variability also limits diagnostic consistency ${ }^{8-10}$. The development of several blood markers such as platelets, hyaluronic acid, type IV collagen, aminotransferase/platelet ratio index (APRI) and algorithm based serum models (fibro Index, FIB-4, and Fibro Test ) have been used but are affected by factors unrelated to the liver.

\section{Elast PQ Ultrasound -a new era in liver diseases assessment:}

A new technique called Elasto PQ uses ultrasound shear wave elastography to provide a noninvasive, reproducible, and easily performed method of assessing liver fibrosis. A special pulse sequence technique that uses existing transducers produces shear waves in tissue and then measures the propagation speed of the waves. Now liver stiffness samples can be acquired during a routine ultrasound examination of the liver. According to a recent study, using share wave elastography may help reduce or avoid conventional liver biopsies ${ }^{11}$. Instead of a costly and painful biopsy procedure, an easy ultrasound exam becomes the routine method to assess liver diseases status.

\section{Performing an Elast PQ examination:}

With increasing fibrosis, the liver becomes stiffer, which can be monitored using shear wave elastography ${ }^{11-12}$ with this technique, during an ultrasound exam, a region of interest (ROI) is placed in an area of the liver taking care not to include large vasculature or biliary structures. An intercostals imaging approach targeting segment 7 or 8 of the liver has been shown to provide more reliable measurements. Serial measurements are taken while the patient suspends respiration and a report is generated. The average of these measurements is then used to estimate the degree of liver stiffness and correlate with a predicted biopsy Metavir score.

Although this technique typically shows strong correlation, there is several confounding factors that may distort results, such as liver inflammation, liver congestion, and biliary obstruction. In some cases, distinguishing normal from very mild diseases may be difficult; some moderate and severe disease may look similar.

\section{Diagnostic capability}

In the study by Sandrin et $\mathrm{al}^{13}$ transient elastography was performed in 91 patients with $\mathrm{HCV}$ related liver disease and a definitive analysis was performed for 67 . In $93 \%$ of cases with F0-F1 fibrosis according to METAVIR elasticity was $5.1 \mathrm{kPa}$. On the other hand, $94 \%$ of cases in F2 stage this value was equal to or higher than $7.6 \mathrm{kPa}$.

The ability of FS to predict fibrosis stage was also assessed in patients confected with HCV. Elastography for diagnosis cirrhosis in this group of patients, when compared to serum markers such as APRI, FIB-4, AST/ALT ratio and platelet count, was higher in a statistically significant way $^{14}$. A recent systematic review by Shaheen et $\mathrm{al}^{15}$ of studies by Castera, Colletta, $\mathrm{Ziol}^{16}$ and ledinghen suggests that diagnostic accuracy is excellent for the detection of cirrhosis associated with $\mathrm{HCV}$, but usefulness is lower for earlier stage. The usefulness of FS has also been recently reported in patients with HBV hepatitis. 
As regards the impact of steatosis on FS measured elasticity, the possibility was initially suggest that, since fat tissue is softer than normal parenchyma, the presence of fatty liver would decrease measurements ${ }^{13}$. However, a letter published by Yoneda et al provided evidence that the correlation between elasticity and fibrosis severity is not modified by steatosis extent ${ }^{17}$. In fact, our own group published an abstract stating that the diagnosis accuracy of FS for the detection of clinical significant fibrosis (F2), advanced fibrosis (F3) and cirrhosis, as measured with Elastography, is higher when compared to APRI and Forn's test in subjects with histologically proven non-alcoholic fatty liver disease $^{18}$.

Regarding cirrhosis, FS has been reported to exhibit a higher power for its exclusion as compared to its prediction. According to Ganne-Carrie et al. a value higher than $14.6 \mathrm{kPa}$ has a Sensitivity of $95 \%$ and Negative Predictive Value of $96 \%$. False negative results resulted in $29 \%$ of cases from the presence of macronodular cirrhosis, and in the remaining $71 \%$ from absent of mild inflammatory activity ${ }^{19}$.

Foucher et al, in turn, in a study with various liver conditions established a number of cutoffs from which several complications secondary to liver cirrhosis would develop, including esophageal varices at 27.5 $\mathrm{kPa}$ and ascites at $49.1 \mathrm{~Pa}^{20}$.

\section{Conclusion :}

In chronic liver diseases patient prognosis and treatment options depend on fibrosis stage, including the potential development of cirrhosis and its complications. Liver biopsy is the most valuable test for liver fibrosis staging; however, given its invasive nature. Noninvasive tests have been recently tried to document liver disease stage. Transient elastograpy, despite limitations and given its relative availability and simplicity, seems to find a place in clinical practice. However, as with serum fibrosis markers, its ability to discriminate between adjacent fibrosis stages is scarce. Its clinical role will likely be greater when specific cutoff points are used for each disease. Elast PQ elastography may offer an ideal way to routinely monitor liver tissue stiffness, and reduce other, more costly and invasive methods of testing.

\section{References :}

1. European Association for the study of the Liver. EASL Clinical Practice Guidelines: management of Hepatitis C virus infection. Journal of Hepatology 2011; 55 (2):245-64.

2. European Association for the study of the Liver. EASL Clinical Practice Guidelines: management of Hepatitis B virus Journal of Hepatology 2012; 57 (1): 265-85.
3. World Health Organization. Viral hepatitis. Report from the Secretariat. Sixty-third World Health Assembly; 2010.

4. Rockey DC, Bissell DM. Noninvasive measures of liver fibrosis. Hepatology 2006:43 (2 Supl I): S113-S120.

5. Barr RG, Ferraiolli G, Palmeri ML, Goodman ZD, Garcia-Tsao G, Rubin J, et al. Elastography assessment of liver Fibrosis : Society of Radiologists in Ultrasound Consensus conference Statement. Radiology 2015; 276:845-61.

6. Bedossa P, Poynard T. An algorithm for the grading of activity in chronic Hepatitis C. The METAVIR cooperative study group. Hepatology 1996; 24(2):289-93.

7. Bravo AA, Sheth SG, Chopra S. Liver biopsy. The New England journal of Medicine 2001; 344(7): 495-500.

8. Cadranel JF, Rufat P, Degos F. Practices of liver biopsy in France: results of a prospective nationwide survey. For the Group of Epidemiology of the French Association for the study of the Liver (AFEF). Hepatology 2000; 32(2):477-81.

9. Maharaj B, Maharaj RJ , Leary WP, et al. Sampling variability and its influence on the diagnostic yield of percutaneous needle biopsy of the liver. Lancet 1986; 1(8480):523-5.

10. Bedossa P, Dargere D, Paradis V. Sampling variability of liver fibrosis in chronic Hepetitis C. Hepatology 2003; 38(6):1449-57.

11. Ferraioli G, Lissandrin Rajbari. Reproducibility and performance of a new point shear wave elastography technique for assessing fibrosis in chronic Hepatitis C. World Journal of Gastroenterology. 2014; 20(16):4787-96.

12. Ferraioli G, Gulizia R, Filice C. Real-time elastography in the assessment of liver fibrosis. AJR Am J Roentgenol. 2007;189:W170.

13. Sandrin L, Fourquet B, Hasquenoph JM, Yon S, Fournier C, Mal F, et al. Transient elastography: A new noninvasive method for assessment of hepatic fibrosis . Ultrasound Med Biol. 2003; 29(12):1705-13.

14. De Ledinghen V, Douvin C, Kettaneh A, Ziol M, Roulot D, Marcellin P, et al. Diagnosis of hepatic fibrosis and cirrhosis by transient elastography in HIV/hepatitis C virus-co infected patients. J, Ac quir Immune Defic Syndr. 2006; 41(2):175-9.

15. Shaheen AAM, Myers RP. FibroScan for the prediction of hepatitis, C- fibrosis: A systematic and review of diagnostic test accuracy. Am J Gastroenterol 2007; 102:2589-600.

16. Ziol M, Handra-Luca A, Kettaneh A, Christidis C, Mal F, Kazemi $\mathrm{F}$, et al. Noninvasive assessment of liver fibrosis by measurement of stiffness in patients with chronic hepatitis C. Hepatology 2005; 41(1):48-54.

17. Yoneda M, Fujita K, Inamori M, Nakajima A, Tamano M, Hiraishi $\mathrm{H}$. Transient elastography in patients with non-alcoholic fatty liver disease (NAFLD), [Letter ], Gut 2007; 56(9):1330-1.

18. Marin-Gabriel JC, De la Cruz J, Ferandez I, Marti AM, Munoz $\mathrm{R}$, Colina F, et al. Prospective comparison between transient elastoghraphy (fibroscan), APRI and Forn's test for assessing liver fibrosis in patients with histological features of NAFLD. J Hepatol 2007; 46:S275

19. Ganne-Carrie N, Ziol M, de Ledinghen V, Douvin C, Marcellin P, Castera L, et al. Accuracy of liver stiffness measurement for the diagnosis of cirrhosis in patients with chronic liver diseases. Hepatology 2006; 44(6):1511-7.

20. Foucher J, Chanteloup E, Vergniol j, Castera L, Bail B, Adhoute $\mathrm{X}$, et al. Diagnosis of cirrhosis by transient elastography (FibroScan): a prospective study, Gut 2006; 55(3): 403-8. 